\title{
Impact of Psychological Distress on Treatment Timeliness in Oncology Patients at a Safety-Net Hospital
}

\author{
Sheshadri Madhusudhana, $\mathrm{MD}^{1,2}$; Michelle Gates, $\mathrm{PhD}^{2}$; Daulath Singh, $\mathrm{MD}^{3}$; Punita Grover, $\mathrm{MD}^{4}$; \\ Mahathi Indaram, $\mathrm{MD}^{5}$; and An-Lin Cheng, $\mathrm{PhD}^{1}$
}

\begin{abstract}
Background: Psychological distress is common in patients with cancer. Distress can affect patients' engagement with treatment. We examined the relationship between psychological distress and treatment timeliness in a sample of adult oncology patients at a safety-net hospital. Methods: A retrospective review was conducted of all patients screened for distress at a first outpatient oncology visit between March 1, 2014, and December 31, $2015(n=500)$. The analytic sample $(n=96)$ included patients with a new cancer diagnosis and a curative-intent treatment plan for lymphoma (stage I-IV), solid tumor malignancy (stage I-III), or head and neck cancer (stage I-IVb). Distress was measured using the Hospital Anxiety and Depression Scale. Using Poisson regression, we determined the effects of depression and anxiety on treatment timeliness. Patient age, sex, race/ethnicity, insurance type, cancer site, and cancer stage were included as covariates. Results: Mean patient age was 54 years. The median treatment initiation interval was 28 days. Clinically significant anxiety was present in $34 \%$ of the sample, and clinically significant depression in 15\%. Greater symptom severity in both anxiety and depression were associated with a longer treatment initiation interval after controlling for demographics and disease factors. The average days to treatment (DTT) was 4 days longer for patients with elevated anxiety scores and for those with elevated depression scores compared with those without. Overall survival was not associated with anxiety, depression, or DTT. Conclusions: In this safety-net patient sample, greater psychological distress was associated with slower time to treatment. As of writing, this is a new finding in the literature, and as such, replication studies utilizing diverse samples and distress measurement tools are needed.
\end{abstract}

J Natl Compr Canc Netw, doi: 10.6004/jnccn.2021.7018 Published online August 11, 2021

${ }^{1}$ University of Missouri-Kansas City School of Medicine and ${ }^{2}$ Truman Medical Centers, Kansas City, Missouri; ${ }^{3}$ Stormont Vail Health, Topeka, Kansas; ${ }^{4}$ University of Cincinnati Medical Center, Cincinnati, Ohio; and ${ }^{5}$ Allegheny General Hospital, Pittsburgh, Pennsylvania.

\section{Background}

Timely treatment is core to maximizing outcomes in patients with cancer. ${ }^{1,2}$ A study of more than 3.6 million patients with cancer demonstrated that longer time intervals between diagnosis and initial treatment were associated with worse survival in stage I colorectal cancers, stage I-III breast and renal cancers, and stage I-II nonsmall cell lung and pancreas cancers. ${ }^{3}$ The same effect has been found in early-stage liver cancer. ${ }^{4}$ Delays in administration of adjuvant therapy has been shown to negatively impact outcomes in stage III colon cancer ${ }^{5}$ and stage I-III breast cancer. ${ }^{6}$ Although a few studies have found a paradoxical relationship of shorter treatment initiation intervals correlating with poorer survival, this likely reflects the preferential initiation of immediate treatment for patients with poorer prognoses and greater symptom burden at diagnosis, such as in the case of advanced lung cancer. ${ }^{7,8}$

Previous research on treatment timeliness has primarily examined sociodemographic and clinical variables. ${ }^{3,9-13}$ Black race, ${ }^{3,9}$ Hispanic ethnicity, ${ }^{9,10}$ non-English language spoken, ${ }^{9,10}$ low educational attainment, ${ }^{11}$ lack of insurance, ${ }^{10}$ noncommercial insurance, ${ }^{13}$ medical comorbidities, ${ }^{9,11,12}$ and asymptomatic presentation at diagnosis ${ }^{11}$ have been identified as risk factors for longer treatment initiation intervals. Safety-net hospitals, by mission or mandate, provide care to a substantial share of vulnerable patients regardless of their ability to pay, according to the National Academy of Medicine (formerly Institute of Medicine). ${ }^{14}$ Our nation's safety-net hospitals have large Medicaid caseloads, and provide substantial amounts of uncompensated care.$^{15}$ As such, patients in these settings may be particularly at risk for long treatment initiation intervals, and subsequently, poorer outcomes. Many oncology clinics have instituted patient navigation programs to facilitate timely care by connecting patients to transportation and financial aid. ${ }^{16}$ Studies suggest that these programs can have positive, albeit modest impacts on treatment timeliness in safety-net oncology settings. ${ }^{16,17}$

Psychological variables have not been well examined as potential correlates of the treatment initiation interval. 
Up-to-date guidelines for the management of psychological distress in oncology have been available from NCCN since 1999. ${ }^{18}$ Evidence indicates that distress is experienced by approximately one-third of patients with cancer; clinically significant psychological symptoms have been found in $35 \%$ of patients presenting to initial oncology visits and in $38 \%$ of patients in a large sample of those attending initial visits, treatment appointments, or follow-up visits. ${ }^{19,20}$ Meta-analysis of 70 published studies estimates that $10 \%$ of patients with cancer have an anxiety disorder and $16 \%$ have major depressive disorder. ${ }^{21}$ However, psychological distress data are lacking for oncology patients treated in the safety-net setting. We identified only one published study quantifying psychological distress in this population. Cimino et $\mathrm{al}^{22}$ used the NCCN Distress Thermometer (as opposed to measuring depression and anxiety) and found that $61 \%$ of patients with cancer in a safety-net setting endorsed clinically significant distress at their initial medical oncology visit.

Psychological distress degrades patients' quality of life and also has implications for treatment course and outcomes. A meta-analysis of data from 76 studies of depression in patients with cancer found that depression was associated with elevated mortality after controlling for relevant clinical variables. ${ }^{23}$ Patient behavior may play a role in mediating the relationship between depression and mortality. A meta-analysis of 12 studies $(n=605)$ demonstrated that medical patients with depression were 3 times more likely to be nonadherent with appointment attendance, taking prescribed medications, and making lifestyle changes compared with patients without depression..$^{24}$ In that analysis, 3 studies included patients with cancer and the remainder included patients with other medical conditions, including rheumatoid arthritis, end-stage renal disease, and angina. Patients with anxiety may be similarly at risk for suboptimal treatment engagement due to fears, phobias, and avoidance of necessary procedures, such as biopsies, surgeries, and imaging.

As of this writing, no published studies have examined a potential relationship between psychological distress and treatment timeliness in oncology. The primary aim of our study was to assess the relationship between psychological distress and the time elapsed between the initial medical oncology visit and the first chemotherapy or chemoradiotherapy (CRT) treatment. Based on the available published data, we expect that anxiety and depression will be associated with longer treatment initiation intervals. A secondary aim of the study was to identify the prevalence of psychological distress among patients newly diagnosed with cancer and determine the treatment initiation interval in a safety-net hospital. Distress data are lacking for safety-net patients. Additionally, the extant literature suggests that treatment initiation intervals may be longer in safety-net hospitals because of the presence of risk factors such as race, ethnicity, education, comorbidities, and insurance status. Finally, we sought to quantify survival outcomes and identify any relationships between distress and survival or treatment timeliness and survival.

\section{Methods}

\section{Study Design and Setting}

We conducted a retrospective cohort study of adult oncology outpatients screened for psychological distress and subsequently treated with curative intent chemotherapy or CRT at a safety-net hospital in the midwestern United States. The study was conducted with approval from the local Institutional Review Board.

\section{Study Sample}

As standard clinical practice, all patients attending their first outpatient medical oncology visit are administered the distress measure. The initial study sample consisted of all patients who completed the screening measure $(\mathrm{n}=500)$ between March 1, 2014, and December 31, 2015. Cases with a new cancer diagnosis and a curative-intent treatment plan including adjuvant chemotherapy, definitive chemotherapy, or definitive CRT were selected for study inclusion. Eligible diagnoses were lymphoma (stage I-IV), solid tumor malignancy after completion of definitive surgical resection (stage I-III), and head and neck cancer (stage I-IVb). Hematologic malignancies including multiple myeloma, indolent lymphoma, and acute leukemia were excluded because their treatment plans are noncurative or require inpatient management. Patients with recurrent disease at presentation were also excluded from the study (Figure 1).

\section{Instruments}

Psychological distress was measured using the Hospital Anxiety and Depression Scale (HADS). ${ }^{25}$ The HADS consists of 14 self-report items assessing affective symptoms of anxiety and depression and yields subscale scores for each. Items use a 4-point (0-3) forced-choice response scale. Subscale scores range from 0 to 21 , with greater scores indicating greater symptom severity. Scores $\geq 11$ on either subscale indicate a probable case of anxiety or depression and reflect clinically significant moderate to severe symptoms. Scores between 8 and 10 indicate mild symptoms, and scores of $\leq 7$ are considered noncases. ${ }^{26}$ A review of 71 published studies utilizing the HADS deemed the instrument to have an empirically supported 2-factor structure, good internal consistency reliability (mean anxiety, $\alpha=0.83$; mean depression, $\alpha=0.82$ ), and good concurrent validity, with correlations among HADS and other similar measures between 0.49 and $0.83 .{ }^{27}$ The HADS assesses emotional and behavioral symptoms to the exclusion of somatic symptoms such as fatigue, 


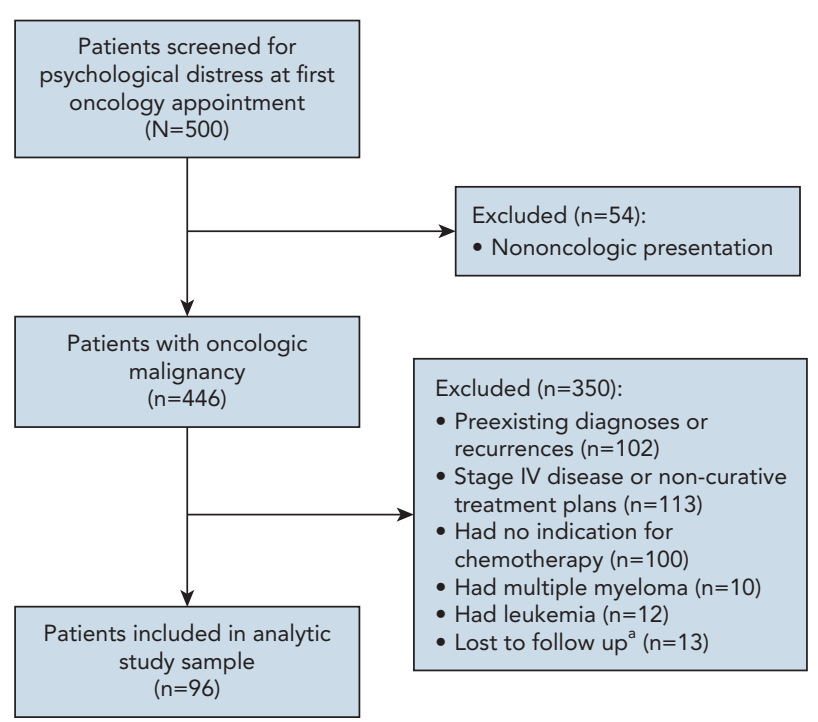

Figure 1. Flowchart of exclusion criteria used to generate analytic study sample.

${ }^{a}$ Includes patients who transferred care to other facilities $(n=3)$ and patients with $>100$ days elapsed between initial office visit and first chemotherapy administration $(n=8)$.

appetite changes, and sleep disturbance. Thus, the meaningfulness of HADS results for this study is enhanced because the impact of physical symptom burden on scores is minimized.

\section{Data Collection and Integrity}

Data were abstracted from electronic health records by study personnel and stored in a secure, deidentified REDCap database. ${ }^{28}$ The following data were abstracted from all records: cancer site and stage; patient age, gender, and race/ethnicity; date of distress screening, distress scores, date of treatment initiation, insurance type, number of days elapsed between the initial medical oncology visit and the first treatment (days to treatment [DTT]), and survival data. The completion rate of the HADS in the study population is not available due to changes in scheduling software used by the institution; however, among the patients who completed the measure and were subsequently included in analysis, none had missing information for any of the variables of interest. The first and second authors of this article (S. Madhusudhana and M. Gates) reviewed all cases for accuracy of chart abstraction. Twelve abstraction errors were corrected prior to analysis (10 errors in date of treatment initiation, 1 error in date of distress screening, 1 error in gender).

\section{Statistical Analyses}

Statistical analyses were conducted using SPSS Statistics, version 24 (IBM Corp) and SAS 9.4 (SAS Institute Inc). Descriptive statistics (ie, frequencies, means, medians, standard deviations, ranges) were used to determine sample characteristics. The primary outcome variable, DTT, was treated as a count response variable. A nonparametric test (Mann-Whitney $U$ test) was used to compare DTT for patients receiving chemotherapy only versus those receiving CRT. Poisson regression analyses were conducted to assess the relationship between DTT and HADS anxiety and HADS depression scores while including age, gender, race, insurance type, and cancer site and stage as covariates in the model. Anxiety and depression scores were treated as interval variables. Cox regression models were applied to assess the relationship between the survival outcome and HADS anxiety and HADS depression scores while including age, gender, race, insurance type, and cancer site and stage as covariates in the model.

\section{Results}

\section{Sample Characteristics}

The final sample consisted of 96 patient cases. Patients ranged in age from 19 to 76 years, with a mean age of 54 years $(\sigma=12)$. Most patients were female $(66.7 \%)$. Approximately half of the patients $(51.0 \%)$ were insured by Medicaid. Approximately half of the patients (51.0\%) were non-Hispanic White and $36.5 \%$ were non-Hispanic Black/African American (Table 1).

\section{Days to Treatment}

Median DTT for the overall sample was 28 days (range, $0-99)$ and the mean DTT was 31 days $(\sigma=22)$. There was no difference in DTT for patients receiving CRT compared with those receiving chemotherapy only (chi-square, 2.82; $P=.09$ ). Median DTT was 26 (range, 2-64) for patients receiving CRT and 28 (range, 0-99) for those receiving chemotherapy only. Increased DTT was associated with older age, breast and lung cancers (vs the "other" disease category), and stage II disease (vs stage IV). Decreased DDT was associated with female gender, Hispanic ethnicity, and race/ethnicity other than Black/African American or White (vs non-Hispanic White), colorectal cancer, gynecologic cancer, and lymphoma (vs the "other" disease category), stage I disease (vs stage IV), and Medicaid and Medicare insurance (vs uninsured) (Table 2).

\section{Psychological Distress}

A total of $34 \%$ of patients obtained clinically significant anxiety scores (scores $\geq 11$ ) and $15 \%$ of patients obtained clinically significant depression scores (scores $\geq 11$ ). The mean scores for both anxiety $(8.4 ; \sigma=4.9)$ and depression $(5.4 ; \sigma=4.7)$ were below the clinically significant cutoff score for the HADS measure (Table 3). There was no difference in anxiety score (chi-square, $0.02 ; P=.89$ ) or depression score (chi-square, $0.001 ; P=.97$ ) for insured patients compared with uninsured patient. Patients enrolled in an 


\section{Table 1. Patient Demographics}

\begin{tabular}{|c|c|}
\hline Characteristic & n (\%) \\
\hline Total, N & 96 \\
\hline \multicolumn{2}{|l|}{ Gender } \\
\hline Female & $64(66.7)$ \\
\hline Male & $32(33.3)$ \\
\hline \multicolumn{2}{|l|}{ Race/Ethnicity } \\
\hline Non-Hispanic White & $49(51.0)$ \\
\hline Non-Hispanic Black/African American & $35(36.5)$ \\
\hline Hispanic & $8(8.3)$ \\
\hline Other & $4(4.2)$ \\
\hline \multicolumn{2}{|l|}{ Insurance status at time of data abstraction } \\
\hline Medicaid & $49(51.0)$ \\
\hline Medicare & $11(11.5)$ \\
\hline Commercial $^{a}$ & $13(13.5)$ \\
\hline Institution discount ${ }^{b}$ & $16(16.7)$ \\
\hline Uninsured & $7(7.3)$ \\
\hline \multicolumn{2}{|l|}{ Disease site/type } \\
\hline Breast & $30(31.3)$ \\
\hline Colorectal & $9(9.4)$ \\
\hline Gynecologic & $16(16.7)$ \\
\hline Head and neck, squamous & $8(8.3)$ \\
\hline Lung, non-small cell & $6(6.3)$ \\
\hline Lymphoma & $9(9.4)$ \\
\hline Other $^{c}$ & $18(19.0)$ \\
\hline \multicolumn{2}{|l|}{ Disease stage } \\
\hline 1 & $14(14.6)$ \\
\hline II & $30(31.3)$ \\
\hline III & $44(45.8)$ \\
\hline $\mathrm{IV}^{\mathrm{d}}$ & $8(8.3)$ \\
\hline \multicolumn{2}{|l|}{ Treatment } \\
\hline Chemotherapy & $62(64.6)$ \\
\hline Chemoradiotherapy & $34(35.4)$ \\
\hline
\end{tabular}

ancludes 2 patients with combination coverage (ie, commercial insurance and Medicare).

${ }^{b}$ Patients who were enrolled in an institution-specific charity discount program; they have no other payer source.

'Includes patients with the following cancers: esophageal $(n=5)$, small cell lung $(n=3)$, pancreas $(n=3)$, anal $(n=2)$, testicular $(n=2)$, urothelial $(n=1)$, cholangiocarcinoma $(n=1)$, sarcoma $(n=1)$.

Includes patients with lymphoma or head and neck cancers only.

institution-specific discount program were categorized as "insured" in the analysis.

\section{Distress and DTT}

Anxiety score was significantly associated with DTT such that higher scores were predictive of a longer treatment initiation interval (relative risk, $1.01 ; P<.05 ; 95 \% \mathrm{CI}$, $1.00-1.02)$. Depression score was also significantly associated with DTT, with higher scores predictive of a longer treatment initiation interval (relative risk, 1.01; $P<.001$; 95\% CI, 1.00-1.02) (Table 2). On average, the treatment initiation interval (DTT) was 4 days longer for patients with significant anxiety symptoms compared with those without elevated anxiety. The average interval was 4 days longer for patients with significant depressive symptoms compared with those without elevated depression (Table 4).

\section{Overall Survival}

Data were collected between March 1, 2014, and December 31,2015 . As of July $1,2020,64 \%$ of patients were alive and $36 \%$ of patients were deceased. Survival was not related to anxiety score (chi-square, $0.91 ; P=.34$ ), depression score (chi-square, $1.95 ; P=.16$ ), or DTT (chi-square, $0.95 ; P=.33)$.

\section{Discussion}

The primary finding of this study was that greater psychological distress at the first medical oncology visit was associated with longer treatment initiation intervals in a sample of patients newly diagnosed with cancer in a safety-net hospital. This is a novel finding; however, it dovetails with previous literature indicating that depression is a risk factor for poorer cancer survival ${ }^{23}$ and is associated with poor adherence to treatment plans in patients with cancer and other medical conditions. ${ }^{24}$ The manifestations of depression (eg, apathy, hopelessness) and anxiety (eg, fear, avoidance) may be the mechanisms by which psychological distress contributes to slower treatment initiation via patient behavior. Because a true experimental design to assess the relationship between psychological distress and treatment timeliness is not practical or ethical, causal interpretations of the current data cannot be definitively made. Additionally, retrospectively collected data are more vulnerable to bias compared with those obtained through prospective study. Nevertheless, a strength of the present correlational study is the temporal relationship between the 2 variables of interest. The measurement of distress occurred prior to the initiation of chemotherapy or CRT treatment, which bolsters the interpretation that distress influenced treatment timeliness as opposed to treatment timeliness influencing distress.

In the current sample, the average DTT was 4 days longer for patients with significant anxiety symptoms and those with significant depressive symptoms compared with patients without these elevated symptoms. There was no relationship between DTT and survival. In a large-scale study of the National Cancer Database, however, risk of mortality in early-stage breast, lung, renal, and pancreas cancers increased by $1 \%$ to $3 \%$ for each week that elapsed between diagnosis and first 


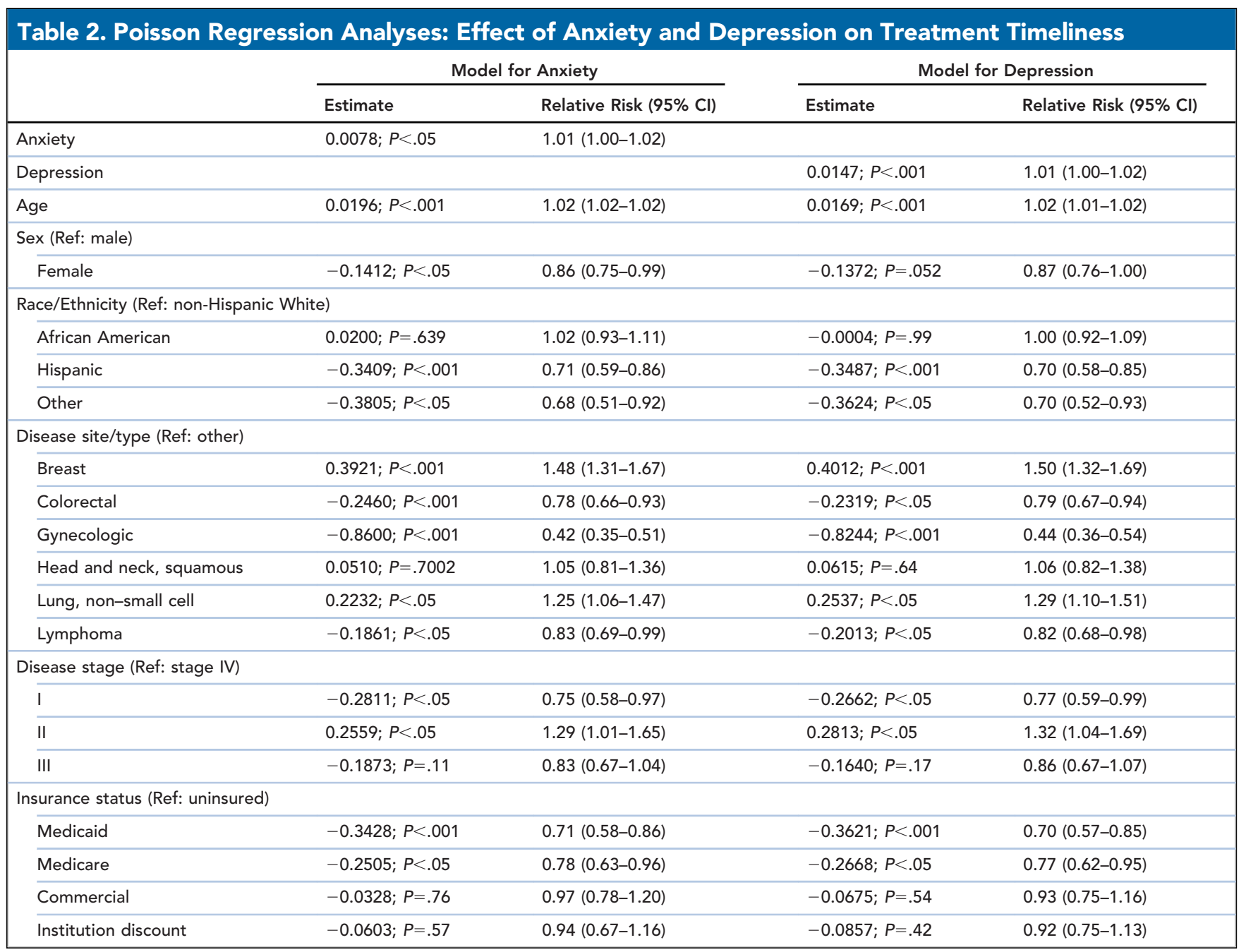

treatment and was particularly pronounced after 6 weeks had elapsed. ${ }^{3}$ In a study of early-stage liver cancer, increased mortality was observed when $>8$ weeks elapsed between diagnosis and treatment. ${ }^{4}$ Mortality risk has also been found to be greater for stage III colon cancer when $>8$ weeks elapsed between surgery and adjuvant chemotherapy. ${ }^{5}$ As such, it is currently unclear if an average delay of 4 days may be clinically meaningful; further research would be helpful in this regard.

A secondary finding of the present study is the descriptive data regarding the prevalence of psychological distress and the length of the treatment initiation interval in the safety-net setting, both of which are lacking in published studies. In this sample, 1 in 3 patients reported anxiety symptoms of at least moderate severity and 1 in 7 reported depressive symptoms of at least moderate severity. In a previous study with a large sample $(n=4,496)$ of patients with mixed diagnoses assessed at the initial medical oncology visit, Zabora et $\mathrm{al}^{20}$ found that $35 \%$ reported clinically significant global distress (a composite of symptoms of anxiety, depression, and somatization). This study was not conducted in the safety-net setting, however, and the sample was $82 \%$ White and $60 \%$ had commercial insurance. Cimino et $\mathrm{al}^{22}$ assessed a mixeddiagnosis sample of patients in a safety-net setting at the initial medical oncology visit and found that $61 \%$ had clinically significant distress. That study used the NCCN Distress Thermometer, which is known to reflect symptoms of anxiety and depression as well as other aspects of distress, including spiritual, physical, and social problems. ${ }^{29}$

In the present study, the median DTT, defined as the number of days elapsed between the initial oncology visit and first chemotherapy or CRT treatment, was 28. In contrast with our study, many studies define treatment timeliness in terms of the number of days elapsed between pathologic diagnosis and first treatment, ${ }^{3,4,8}$ or in the case of adjuvant treatment initiation, the interval is defined as the number of days elapsed between 


\begin{tabular}{|ll|}
\hline $\begin{array}{l}\text { Table 3. Psychological Distress: HADS Scores } \\
(\mathbf{N = 9 6 )}\end{array}$ & $\mathrm{n}(\%)$ \\
\hline HADS Score & $45(46.9)$ \\
\hline \begin{tabular}{l} 
Anxiety \\
\hline Noncase (score $\leq 7)$
\end{tabular} & $18(18.8)$ \\
\hline Mild (score 8-10) & $33(34.4)$ \\
\hline Moderate-severe (score $\geq 11)$ & $67(69.8)$ \\
\hline Depression & $14(14.6)$ \\
\hline Noncase (score $\leq 7)$ & $15^{\mathrm{a}}(15.6)$ \\
\hline Mild (score 8-10) & \\
\hline Moderate-severe (score $\geq 11)$ &
\end{tabular}

Abbreviation: HADS, Hospital Anxiety and Depression Scale.

${ }^{a}$ All patients with depression scores in the moderate-severe range also had anxiety scores in the moderate-severe range.

surgery and administration of adjuvant therapy. ${ }^{5,6}$ Khorana et $\mathrm{al}^{3}$ found a median time between diagnosis and first treatment of 29 days in a sample of $>700,000$ patients diagnosed with cancer between 2012 and 2013. In studies of adjuvant therapy initiation after surgery for breast and colon cancers, intervals of 31 to 60 days $^{6}$ and 35 to 60 days, ${ }^{5}$ respectively, were most common. Due to variation in how treatment timeliness is operationalized, the DTT observed in the current sample cannot be directly compared with those reported in this previous literature; however, it does not appear to be significantly divergent.

Previous studies have found Black race, Hispanic ethnicity, lack of English fluency, low education, medical comorbidities, and lack of insurance to be associated with longer treatment initiation intervals. Individuals with these characteristics are overrepresented in the safety-net settings and patients seen in safety-net hospitals are likely to experience transfers of care between institutions prior to initiation of treatment. ${ }^{30}$ Protective factors against delay, however, are also more likely to be prevalent in safety-net hospitals, such as patient navigation programs ${ }^{16,17}$ and the ability of uninsured patients to bypass requirements for insurance prior authorization.

The observed relationship between distress and treatment timeliness is a new finding in a specific population (safety-net), and as such, replication studies using diverse samples and distress measurement tools are needed to determine the prevalence and generalizability of this effect. Patient behavior is the proposed mechanism through which depression and anxiety may be impacting treatment timeliness. Future studies can build on the present findings by quantifying relevant patient behavior and including those variables in analyses. Limitations of the current study include its use of a heterogeneous sample of modest size. At the time distress was assessed, some patients had already completed definitive surgical
Table 4. Number of DTT by HADS Score

\begin{tabular}{|lll|}
\hline & $\mathbf{n}$ & $\begin{array}{l}\text { DTT } \\
\text { Mean [SD] }\end{array}$ \\
\hline Anxiety & 33 & $34.0[23.0]$ \\
\hline Elevated (score $\geq 11)$ & 63 & $29.7[20.8]$ \\
\hline Not elevated (score $\leq 10)$ & & \\
\hline Depression & 15 & $34.7[22.6]$ \\
\hline $\begin{array}{l}\text { Elevated (score } \geq 11) \\
\text { Not elevated (score } \leq 10)\end{array}$ & 81 & $30.5[21.4]$ \\
\hline Depression and anxiety & $15^{\mathrm{a}}$ & $34.7[22.6]$ \\
\hline Both scales elevated (scores $\geq 11)$ & 81 & $30.5[21.4]$ \\
\hline Neither scale elevated (scores $\leq 10)$ & & \\
\hline
\end{tabular}

Abbreviations: DTT, days to treatment; HADS, Hospital Anxiety and Depression Scale.

${ }^{a}$ All patients with elevated depression scores also had elevated anxiety scores.

resection and some had yet to initiate their primary treatment; the potential relevance of this for the relationship between distress and timeliness of subsequent chemotherapy (or CRT) is an empirical question requiring further investigation. The modest sample size in the current study may explain the lack of statistical relationship between treatment timeliness and survival in the current sample, which is inconsistent with previous research. Although the present study accounted for several important covariates, medical comorbidities and symptom burden were not included, both of which have been linked to treatment timeliness in previous studies.

\section{Conclusions}

This retrospective study provides preliminary evidence that psychological distress may have a meaningful impact on treatment timeliness in oncology. Further studies aimed at replicability and generalizability of this effect are needed to determine the implications for clinical management. If the findings are reproduced, they represent the identification of a modifiable barrier to treatment timeliness in oncology.

Submitted February 10, 2020; final revision received January 14, 2021 accepted for publication January 28, 2021.

Published online August 11, 2021.

Disclosures: The authors have disclosed that they have no financial interests, arrangements, or affiliations with the manufacturers of any products discussed in this article or their competitors.

Author contributions: Study concept: Madhusudhana. Data collection: Madhusudhana, Singh, Grover, Indaram. Data entry: Singh, Grover, Indaram. Data analysis: Madhusudhana, Gates, Singh, Grover, Indaram. Manuscript preparation: Madhusudhana, Gates. Statistical analyses: Cheng.

Correspondence: Sheshadri Madhusudhana, MD, Hospital Hill Center, Truman Medical Centers, 2310 Holmes Street, Kansas City, MO 64108. Email: Madhusudhanas@umkc.edu 


\section{References}

1. Williams F. Assessment of breast cancer treatment delay on prognosis and survival: a look at the evidence from systematic analysis of the literature. J Cancer Biol Res 2015;3:1071.

2. Huang J, Barbera L, Brouwers M, et al. Does delay in starting treatment affect the outcomes of radiotherapy? A systematic review. J Clin Oncol 2003;21:555-563.

3. Khorana AA, Tullio K, Elson P, et al. Time to initial cancer treatment in the United States and association with survival over time: an observational study. PLoS One 2019;14:e0213209.

4. Tsai WC, Kung PT, Wang YH, et al. Influence of the time interval from diagnosis to treatment on survival for early-stage liver cancer. PLoS One 2018;13:e0199532.

5. Gao $P$, Huang $X Z$, Song $Y X$, et al. Impact of timing of adjuvant chemotherapy on survival in stage III colon cancer: a population-based study. BMC Cancer 2018;18:234.

6. Chavez-MacGregor M, Clarke CA, Lichtensztajn DY, et al. Delayed initiation of adjuvant chemotherapy among patients with breast cancer. JAMA Oncol 2016;2:322-329.

7. Diaconescu R, Lafond C, Whittom R. Treatment delays in non-small cell lung cancer and their prognostic implications. J Thorac Oncol 2011;6: 1254-1259.

8. Myrdal G, Lambe M, Hillerdal G, et al. Effect of delays on prognosis in patients with non-small cell lung cancer. Thorax 2004;59:45-49.

9. Halpern MT, Holden DJ. Disparities in timeliness of care for U.S. Medicare patients diagnosed with cancer. Curr Oncol 2012;19:e404-413.

10. Jaiswal K, Hull M, Furniss AL, et al. Delays in diagnosis and treatment of breast cancer: a safety-net population profile. J Natl Compr Canc Netw 2018;16:1451-1457

11. Zarcos-Pedrinaci I, Fernández-López A, Téllez T, et al. Factors that influence treatment delay in patients with colorectal cancer. Oncotarget 2017; 8:36728-36742.

12. Shen $S$, Hung $Y$, Kung $P$, et al. Factors involved in the delay of treatment initiation for cervical cancer patients: a nation-wide population based study. Medicine (Baltimore) 2016;95:e4568.

13. Kan CK, Qureshi MM, Gupta A, et al. Risk factors involved in treatment delays and differences in treatment type for patients with prostate cancer by risk category in an academic safety net hospital. Adv Radiat Oncol 2017; 3:181-189.

14. Institute of Medicine. America's Health Care Safety Net: Intact But Endangered. Washington, DC: National Academies Press; 2000.

15. Popescu I, Fingar KR, Cutler E, et al. Comparison of 3 safety-net hospital definitions and association with hospital characteristics. JAMA Netw Open 2019;2:e198577.
16. Haideri NA, Moormeier JA. Impact of patient navigation from diagnosis to treatment in an urban safety net breast cancer population. J Cancer 2011;2: 467-473.

17. Miller MK, Orangio GR, Kahanda R, et al. Diagnostic and treatment delays of colorectal cancer in a safety-net hospital: the influence of a patient navigator and a colorectal cancer pathway on a high-risk population. Poster presented at American College of Surgeons Clinical Congress 2017 . October 22-26, 2017; San Diego, California.

18. Riba MR, Donovan KA, Andersen B, et al. NCCN Clinical Practice Guidelines in Oncology: Distress Management, Version 2.2020. Accessed April 24, 2020. To view the most recent version, visit NCCN.org

19. Carlson LE, Angen M, Cullum J, et al. High levels of untreated distress and fatigue in cancer patients. Br J Cancer 2004;90:2297-2304.

20. Zabora J, BrintzenhofeSzoc K, Curbow B, et al. The prevalence of psychological distress by cancer site. Psychooncology 2001;10:19-28.

21. Mitchell AJ, Chan M, Bhatti H, et al. Prevalence of depression, anxiety, and adjustment disorder in oncological, haematological, and palliative-care settings: a meta-analysis of 94 interview-based studies. Lancet Oncol 2011; 12:160-174.

22. Cimino $T$, Said $K$, Safier $L$, et al. Psychosocial distress among oncology patients in the safety net. Psychooncology 2020;29:1927-1935.

23. Pinquart $M$, Duberstein PR. Depression and cancer mortality: a metaanalysis. Psychol Med 2010;40:1797-1810.

24. DiMatteo MR, Lepper HS, Croghan TW. Depression is a risk factor for noncompliance with medical treatment: meta-analysis of the effects of anxiety and depression on patient adherence. Arch Intern Med 2000;160: 2101-2107.

25. Zigmond AS, Snaith RP. The hospital anxiety and depression scale. Acta Psychiatr Scand 1983;67:361-370.

26. Snaith RP. The Hospital Anxiety And Depression Scale. Health Qual Life Outcomes 2003;1:29.

27. Bjelland I, Dahl AA, Haug TT, et al. The validity of the Hospital Anxiety and Depression Scale. An updated literature review. J Psychosom Res 2002;52: 69-77.

28. Harris PA, Taylor R, Thielke R, et al. Research electronic data capture (REDCap) - a metadata-driven methodology and workflow process for providing translational research informatics support. J Biomed Inform 2009;42:377-381.

29. Hoffman BM, Zevon MA, D'Arrigo MC, et al. Screening for distress in cancer patients: the NCCN rapid-screening measure. Psychooncology 2004;13:792-799.

30. Shah JP, Valdes M, Rockey DC. Transferred and delayed care of patients with colorectal cancer in a safety-net hospital system-manifestations of a distressed healthcare system. J Gen Intern Med 2012;27:1142-1149. 